\title{
GALE A PSORERGATES MUSCULINUS (Michael 1889) (ACARI ; MYOBIIDE) CHEZ LES MULOTS APODEMUS SYLVATICUS (Linné 1758) DU MASSIF DU CAROUX (HERAULT)
}

\author{
Par Jean-A. RIOUX et Yves-J. GOLVAN
}

Le genre Psorergates a été créé en 1883 par J. B. Tyrrell pour Psorergates simplex Tyrrell 1883, parasite de la Souris (Mus musculus Linné 1758) au Canada. Mais, selon M. G. Neumann (1893), cet Acarien aurait été vu dès 1857 par Gerlach qui l'a sommairement décrit et figuré. En 1889, sous le nom de Goniomerus musculinus, A. D. Michaël signale chez le Campagnol [Microtus agrestis (Linné 1761) ] une forme voisine qui est rapportée au genre Psorergates par A. C. Oudemans en 1906.

L'espèce $P$. simplex Tyrrell 1883 a été retrouvée en 1886 , en Italie, chez la Souris par G. P. Piana (sub nom. Sarcoptes musculus). En 1892, M. G. Neumann l'identifie en France chez Mus musculus et Microtus arvalis (Pallas 1778). Cet auteur, qui a eu l'opportunité de comparer ses préparations à celles de Michaël, sépare, de façon formelle, l'espèce de Tyrrell de celle de Michaël. La seconde présente, en effet, "sur le coude du second article 》 des quatre paires de pattes, «un fort crochet recourbé en-dessous ». Depuis ces travaux, une troisième espèce a été décrite dans le genre : Psorergates ovis Womersley 1941, parasite des Moutons d'Australie.

$P$. simplex a été revu en Grande-Bretagne, chez $M$. agrestis, par L. W. Sambon (1928), qui a discuté des rapports possibles entre les parasitoses et les épithéliomas cutanés et mammaires des Muridés, puis en Amérique du Nord par R. J. Flynn et B. N. Jaroslow (1956) chez la Souris.

Au cours d'une prospection épidémiologique dans le Massif du Caroux (Hérault), menée de décembre à mai 1961, nous avons piégé un certain nombre de Mulots porteurs de gales sarcoptiques à Psorergates musculinus (Michaël 1889) [K. Samsinak det. (1)].

(1) Au professeur K. Samsinak, de Prague, qui a bien voulu nous dèterminer cet Acarien, nous adressons nos vifs remerciements. 
Les adultes sont en effet pourvus d'un fort crochet chitineux sur le « coude» du second article des pattes. Ces crochets existent chez les adultes des deux sexes (fig. 1).

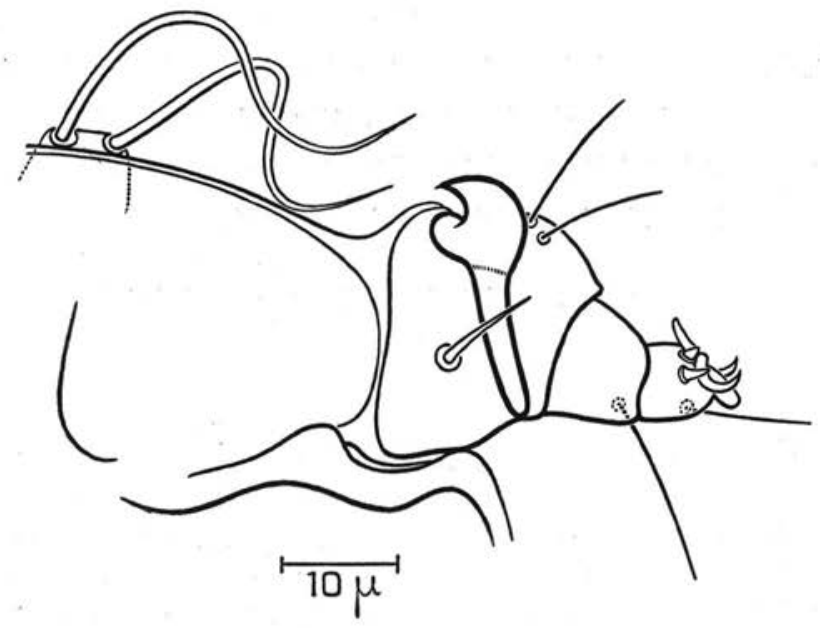

FIG. 1. - Patte postérieure: crochet chitineux caractéristique de Psorergates musculinus

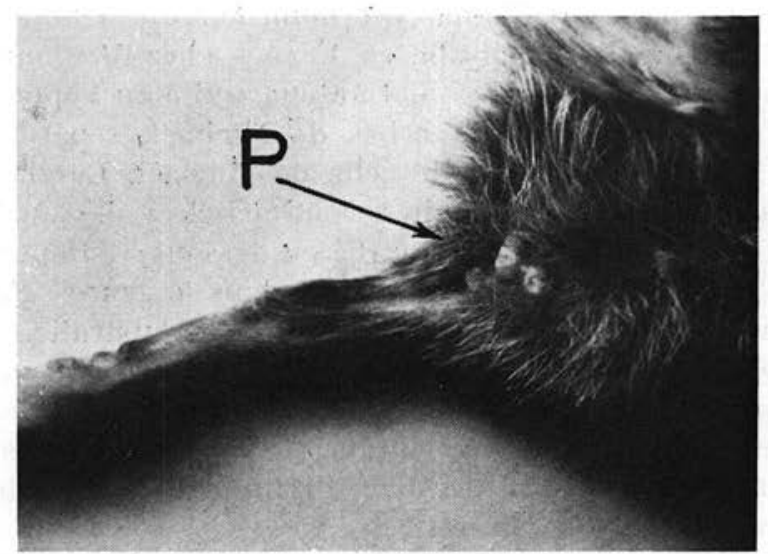

FIg. 2. - Lésions pseudo-papillomateuses (P) sur la racine de la cuisse

Du point de vue macroscopique, les lésions se présentent sous deux aspects. A la phase d'état (fig. 2), elles réalisent le type d'un pseudo-papillome verruqueux, de couleur chamois, sessile, de la 
taille d'un grain de mil à celle d'un gros pois, à « lit» enchâssé profondément dans le derme, à sommet déchiqueté et fissuré. L'examen attentif montre qu'il s'agit, en fait, d'un squame-croûte papyracée comprenant plusieurs lames épidermiques superposées. Le siège de ces « verrues » est variable (pattes, tête, thorax, abdomen) et leur nombre peut être supérieur à dix. La curette parvient assez difficilement à désinsérer la masse papillomateuse, très adhérente au tégument, et laisse après elle un cratère hémorragique profond.

La lésion évolue ainsi de quelques semaines à quelques mois, puis s'élimine spontanément en laissant une cupule faviforme, sèche, à bords jaunâtres.

Au microscope, la lésion apparaît formée de boyaux cornés pseudo-kystiques fermés en cul-de-sac et bourrés d'Acariens à tous les stades de développement.

A notre connaissance, Psorergates musculinus (Michaël 1889) n'a jamais été signalé en France. A podemus sylvaticus constitue d'autre part un hôte nouveau.

\section{BiBLIOGRAPHIE}

Carter (H. B.), 1941. - A skin disease of sheep due to an ectoparasitic mite, Psorergates ovis Womersley 1941. Austral. Veter. Jl., XVII (1), 193-201 (fig. 1-6).

Dubinin (V. B.), 1955. - Acariens de la Gale (Acariformes, Sarcoptidae) et Gales des Mammifères sauvages (en russe). Zool. Zhl., XXXIV (6), 1189-1202 (fig. 1-7).

FLYNN (R. L.), 1959. - Follicular Acariasis of Mice caused by Psorergates simplex successfully treated with Aramite. Americ. Jl. Veter Resch., XX $\left(n^{\circ}\right.$ 74), 198-200 (fig. 1-4).

- et JAROsLow (B. N.), 1956. - Nidification of a mite (Psorergates simplex Tyrrell 1883: Myobiidae) in the skin of Mice. Jl. Parasitol., XLII (1), 49-52 (fig. 1-8).

Gerlach, 1857. - Krätze und Rãude, Hirschwald. Berlin, pp. 178.

Michael (A. D.), 1889. - On some unrecorded Parasitic Acari found in Great Britain. Jl. Linnean Soc. (Zoolog.), XX, 400-406 (1 pl.).

Neumann (M. G.), 1893. - Sur un Acarien (Psorergates simplex Tyrrell) de la souris. Bull. Soc. Hist. Natur. Toulouse, XXVII, 13-22 (1 pl.).

Oudemans (A. C.), 1906. - Révision des Chélétinés. Mém. Soc. Zoolog. France, XIX, 36, 218 (+ fig. 1-66).

Piana (G. P.), 1886. - Cisti cutanee contenenti acari nei topi. Annuar. Ann. Scolast., 1885-1886 (R. Scuola super. med. veter. Milano), I, 122-127 (pl. 1, fig. 8).

SAmbon (L. W.), 1928. - Psorergates simplex as a possible factor of epithelioma of the skin and mammary glands in Murids. Ann. Trop. Med. Parasitol., XXII, 133-136 (fig. 1-2).

Tyrrell (J. B.), 1883. - On the occurence in Canada of two species of Parasitic mites. Proceed. Canadian. Instit. Toronto, I, 332-343 (pl. III-IV).

Instituts de Parasitologie des Facultés de Médecine de Montpellier et de Paris 